\title{
Candidaemia: risk factors and validity of tools to predict risk of Candidaemia in intensive care unit patients in Teaching Hospital Karapitiya
}

\author{
H Thabrew ${ }^{1}$, A Nagahawatte ${ }^{1}$, G B Wijayaratne ${ }^{1}$
}

\begin{abstract}
Background The ability to predict candidaemia gives a significant advantage to the clinician by enabling preemptive treatment to reduce mortality.

Objectives To identify the prevalence, risk factors, and to validate candida colonization index $(\mathrm{Cl})$, candida score (CS) to reliably predict the development of candidaemia in the identified study sample.

Methods Cross sectional analytical study of consecutive admissions fulfilling inclusion criteria to intensive care units (ICUs) of Teaching Hospital Karapitiya from $1^{\text {st }}$ December 2015 to $30^{\text {th }}$ March 2016. Candida colonization of non-blood body sites was measured by culture on admission and repeated every $3^{\text {rd }}$ day until discharge from ICU, death or development of candidaemia. $\mathrm{Cl}$, corrected $\mathrm{Cl}(\mathrm{CCl})$, and $\mathrm{CS}$ were calculated for the study patients.
\end{abstract}

Results Candida colonization was found in 100 (65.7\%) patients out of 152 patients screened. The presence of sepsis, gastro-intestinal infection or surgery, central venous line colonization, higher density of rectal, oral and urine colonization was significantly different among the infected and non-infected groups. Change of species of colonizing candida was also a significant new risk factor found in the study. All the indices and scores had excellent sensitivity and negative predictive values. However none of the scores had good specificity except for $\mathrm{CCl}$, which was $94 \%$.

Conclusions A combination of $\mathrm{Cl}, \mathrm{CCl}$ and $\mathrm{CS}$ formulated for the study sample could reliably predict candidaemia.

Ceylon Medical Journal 2018; 63: 169-173

DOI: http://doi.org/10.4038/cmj.v63i4.8767

\section{Introduction}

Candida is a commensal yeast which is the commonest cause of invasive fungal infections throughout the world, with an incidence rate of 2 to 14 per 100000 in population based studies [1,2]. Advancement of medicine with transplantations, invasive devices and procedures, antibiotic use, and infectious epidemics like HIV has resulted in an ever increasing cohort of candidates susceptible to candida and has led to the rise of candidaemia throughout the world [3]. An alarming rise of candidaemia rates, with associated mortality, has occurred in the recent past. The risk factors for candidaemia vary with the local environment, hygiene and available facilities which necessitate customized studies to identify the risk factors [4]. The main reasons for the high mortality due to candidaemia are delay in initiation of antifungal therapy, treatment with an ineffective antifungal, inadequate dose or duration of treatment, or no treatment at all [3].

Pittet et al in 1994 formulated the candida colonization index (CI). It is calculated by dividing the number of sites of colonization by the total number of sites cultured. Corrected CI (CCI) was calculated by multiplying the Candida CI by the ratio of heavily colonized sites to the number of sites positive. This formula has been used successfully in prediction of candidaemia in critically ill surgical patients. A meta-analysis of studies done in different hospitals in medical and surgical units was published by the same authors in 2014 which revealed the success of prediction of candidaemia by CI [5].

Candida score (CS) was formulated by addition of clinical status of the patient, by Leon et al in 2006. The score gave 1 mark each for multi-focal colonization, parenteral nutrition, and surgery at intensive care unit (ICU) admission and 2 marks for sepsis. A score of more than 2.5 accurately predicted patients who will benefit from antifungal treatment [6].

Studies done by Eggimann in 2014 show that CS was more accurate than CI in predicting candidaemia [7]. Similar studies on candida colonization have not been done in Sri Lanka to identify and to validate the candida colonization index or candida score for Sri Lankan population, which will help to predict the risk of candidaemia in each patient.

${ }^{1}$ Department of Microbiology, Faculty of Medicine, University of Ruhuna, Galle, Sri Lanka.

Correspondence: HT e-mail: <harshanipj@gmail.com>. Received 13 August 2018 and revised version accepted 03 October 2018

This is an open-access article distributed under the terms of the Creative Commons Attribution License, which permits unrestricted use, distribution, and reproduction in any medium, provided the original author and source are credited. 
The aim of this study was to identify the prevalence and risk factors of candidaemia in ICU patients, and to validate the known indicators CI, CCI, CS to predict the risk of candidaemia in the study patients.

\section{Methods}

The study was conducted as a cross sectional analytical study in ICUs of Teaching Hospital Karapitiya. Patients with ICU exposure with and without candidaemia were compared to identify the risk factors and ability of the CI, CCI and CS to predict candidaemia.

Mixed population of medical and surgical patients admitted to main and emergency treatment unit ICUs, and paediatric neonatal ICUs. Oncology ICU admitted patients who underwent oncological surgery. All patients admitted to the above ICUs during the study period who gave informed consent and stayed for more than 24 hours in the ICU with candida colonization were enrolled in the study. Patients who were already on antifungal therapy was excluded. The study was conducted for a period of 4 months starting from 1st December 2015. Ethics approval for the study was granted by the Ethics Review Committee of the Faculty of Medicine, University of Ruhuna, Sri Lanka.

Candida colonization was diagnosed by detection of growth of candida in a minimum of two specimens on the same day or consecutive specimen collection days. Decision for blood culture was taken by the attending clinicians based on the clinical status of the patient. Candidaemia was diagnosed by isolation of candida from minimum of two specimens of peripheral blood culture. Patients were categorized as infected if they had candidaemia and non-infected if they did not have candidaemia.

Swabs from the oropharynx, rectum and insertion port of central lines were collected along with a urine sample every third day from admission. Additionally, fluid from abdominal drains, intercostal drains were obtained when available. Specimen collection was done only by the principal investigator according to the standard operating procedures and it was continued until discharge from ICU, death or development of candidaemia. Data collection was done using an investigator administered questionnaire which collected details of risk factors which were comorbidities, surgical procedures, antibiotic therapy etc.

Specimens were inoculated on candida chromagar. The growth was categorized as scanty, moderate or heavy. Patients were categorized as heavily colonized if they had more than $3+$ growth in rectal and oral swabs and more than 105 colony forming units/ml growth in urine cultures. The isolates were further identified by morphology on corn meal agar and carbon assimilation pattern. Antifungal sensitivity testing was done on isolates for fluconazole and voriconazole by Clinical and Laboratory Standards Institute (CLSI) method (M44A2), and amphotericin according to manufacturer's recommendations. Quality control of AFST was done according to CLSI-M44-A2 recommendations.

Several patients with rising candida colonization and fever not responding to antibiotics were started on fluconazole empirically. They were included in the noninfected group in data analysis and not removed from analysis as it will bias the results.

Sample sizes required for the study for colonized group and infected groups were determined using the equation for the required sample size to estimate a mean value of CCI [9]. A study conducted by Pittet et al in 1994 to calculate mean CCI for colonized and infected groups found a standard deviation of 0.12 for the colonized patients and 0.1 for the infected patients.

$\mathrm{n}=$ Sample number

$\sigma=$ Assumed Standard deviation

$\mathrm{Z}=5 \%$ significance level

$\mathrm{d}=$ Precision

$$
\mathrm{n}=\frac{\mathrm{Z}^{2} \sigma^{2}}{\mathrm{~d}^{2}}
$$

The calculation accordingly gave a value of 61 subjects for the colonized group with precision of 0.03 . For the infected group 0.05 precision level was used due to the rarity of the condition and the limited time of the study. Thus, the required sample size for the infected group was 16. Sample collection was extended to collect the maximum number of infected patients. Only 10 infected patients were found during the study period after extending the colonized group to include 100 patients.

Data was analysed using SPSS software version 20. Patients with candida colonization without infection was compared with patients with candida colonization with infection to identify the risk factors with significant differences. Fisher's exact was used in the analysis and a $p$ value of $<0.05$ was considered significant.

\section{Results}

Study sample included 152 patients. Two adult patients were not included in the study due to severe injuries with pelvic and facial fractures as they were not stable to obtain screening cultures. Four extreme premature neonates were excluded from the study due to instability of their vital functions during handling. The number of patients without candida colonization was 44 . The non-infected group had 90 patients while the infected group had 10 patients.

Candida colonization was found in 100 patients $(65.7 \%)$. Most (40\%) of the patients in the infected group were from more than 65 years age group and none of the patients were from less than 1 month old age group.

Heavy colonization was seen only in rectum, oral cavity and urine. Other cultured sites did not have heavy colonization. 
Table 1. Demographic details of the study participants

\begin{tabular}{lll}
\hline & Infected & Non Infected \\
\hline Total & 10 & 90 \\
Male sex & $7(70 \%)$ & $55(61 \%)$ \\
Age range & 2 months to & 2 days to \\
& 80 years & 83 years \\
Distribution in ICUs & & \\
Main ICU & $5(50 \%)$ & $40(44 \%)$ \\
ETC ICU & $2(20 \%)$ & $22(24 \%)$ \\
PICU & $3(30 \%)$ & $18(20 \%)$ \\
NICU & $0(0 \%)$ & $8(9 \%)$ \\
OICU & $0(0 \%)$ & $2(2 \%)$ \\
\hline
\end{tabular}

Table 2. Description of candida colonization

\begin{tabular}{llll}
\hline & \multicolumn{1}{c}{ Infected } & Non-infected \\
\hline Oral colonization & - Total & $9(90 \%)$ & $79(87 \%)$ \\
& Heavy & $6(60 \%)$ & $21(23 \%)$ \\
Rectal colonization & - Total & $10(100 \%)$ & $66(73 \%)$ \\
& Heavy & $7(70 \%)$ & $15(16 \%)$ \\
Urine colonization & - Total & $4(40 \%)$ & $18(20 \%)$ \\
& Heavy & $5(50 \%)$ & $15(16 \%)$ \\
\hline
\end{tabular}

Colonizing candida species changed during the ICU stay in 6 infected and 14 none infected patients and it was statistically significant in the two groups $(\mathrm{p}=0.004)$. However no regularity was noticed for this change of colonizing species of candida.

Presence of sepsis $(\mathrm{p}=0.006)$, presence of a gastrointestinal infection or surgical procedure $(\mathrm{p}=0.014)$, central line colonization with candida $(\mathrm{p}=0.001)$, heavy rectal $(\mathrm{p}=0.001)$, oral $(\mathrm{p}=0.022)$ and urine $(\mathrm{p}=0.025)$ colonization and change of colonizing species of candida $(\mathrm{p}=0.004)$ was significantly different among the infected and noninfected groups. None of the other risk factors like age more than 65 , male gender, chronic kidney disease, diabetes, administration of total parenteral nutrition, immunosuppression, chemotherapy, neutropenia, administration of steroids had significant difference between infected and non-infected group. Maximum recorded CI of $\geq 0.5$, CCI of $\geq 0.4$ and a CS of $\geq 3$ can be considered in the prediction of candida blood stream infection.

Table 3. Comparison of sensitivity, specificity, positive predictive value (PPV) and negative predictive value (NPV) of the tests

CS- candida score, MaxCl- maximum $\mathrm{Cl}$

\begin{tabular}{lllll}
\hline Parameter & Sensitivity & Specificity & $P P V$ & $N P V$ \\
\hline MaxCI $(\geq 0.5)$ & $80 \%$ & $77.7 \%$ & $28.5 \%$ & $97.2 \%$ \\
MaxCI $(\geq 0.6)$ & $80 \%$ & $78.8 \%$ & $32 \%$ & $98.6 \%$ \\
CCI & $80 \%$ & $94.4 \%$ & $61.5 \%$ & $97.7 \%$ \\
CS & $100 \%$ & $47.7 \%$ & $17.5 \%$ & $100 \%$ \\
\hline
\end{tabular}

\section{Discussion}

Candida colonization percentage during ICU stay in this study sample was $69.4 \%$. Colonization percentage of our population was similar to an Australian study done by Lau et al, (10) on a mixed sample of medical and surgical patients and an Italian study conducted by Caggiano et al, (11) on neurological patients admitted to ICU.

A change of colonizing species of candida during ICU stay was observed in $60 \%$ of the infected patients whereas it was only $15.5 \%$ in the non-infected group. The difference was statistically significant $(\mathrm{p}=0.004)$ and none of the previous studies had similar observations. This change could be due to replacement of the patient's own flora with the hospital flora or could be due to exposure of the patients to risk factors which may predispose them to develop this change. None of our patients were on antifungals during the specimen collection period. Further studies are required to identify the cause for this change which could be important in the management of these patients.

Central line colonization with candida, presence of gastro-intestinal surgery or infection, sepsis, heavy colonization in rectum, oral cavity and urine were significant risk factors for the development of candida blood stream infection in our study.

The NEMIS study done on surgical ICU patients in 2001 showed that presence of a central line, haemodialysis, undergoing surgery were significant risk factors for candidaemia. Multi-site candida colonization was not found to be a significant risk factor in this study (12). This was a study done only on surgical patients although it was a multicentre large prospective study and was the only study which described that multisite colonization was not a significant risk factor in the development of candidaemia.

The study done by Leon et al (6) showed that duration of stay in ICU, surgery at admission to ICU, total parenteral nutrition, haemodialysis, sepsis and multisite candida colonization were significant risk factors for the development of candidaemia. This study was conducted in 73 medical and surgical ICUs of Spain by the EPCAN project.

Maximum CI with a cut-off level of 0.6 identified $80 \%$ of infected patients and increased the specificity, positive predictive value and negative predictive value slightly. CS had excellent sensitivity and negative predictive value in our study. Therefore selecting patients who are above the cut-off limit of both scores CCI and CS for the prediction of candidaemia will help in identifying patients at risk of candidaemia for the clinician.

Maximum CI, CCI and CS were all significantly different among infected and non-infected groups of patients ( $\mathrm{p}=0.000,0.000$ and 0.02 respectively). Sensitivity and specificity of the corrected CI was $100 \%$ in one of 
these earliest studies (8). However results of studies done elsewhere have been variable as shown in the review done by the same authors who formulated the CI [5]. These studies showed that although sensitivity and negative predictive value in surgical patients is $100 \%$ it is $75 \%$ and $94 \%$ in the medical ICU respectively. However, no study done up to date has been successful in the correct prediction of candidaemia with $100 \%$ specificity and positive predictive value.

Some patients who were pre-emptively treated with fluconazole were also included in the non-infected group of patients which could affect the reduction in the specificity and positive predictive value of this sample. The low sensitivity of blood culture in detection of candidaemia has to be considered. A study done in 2012 by the ESCMID study group showed that, if blood cultures were taken properly in a set of six for anaerobic and aerobic incubation, sensitivity of blood culture in detection of candidaemia is 50-75\% [13]. This was not routinely done in our ICUs where only a single blood culture is taken at a time. The authors further recommend that the sensitivity can be improved up to $80 \%$ by combining serial Mannan antigen and anti-Mannan antibody detection with the advantage of results available in an average of 6 days prior to blood culture becoming positive. However cost and availability is a problem for this test.

CS was formulated for non-neutropaenic patients which makes it more suitable for our study sample as only two were neutropaenic. CS gave the best sensitivity and negative predictive value for our sample of patients.

A prospective multi-centre study [14] done in Spain, Argentina and France also showed a significant negative predictive value of the CS. However the specificity was $66.2 \%$ for the CS. Invasive infection was not reported in patients with a CS below 3 . Ahmed et al [15] in their review stressed the importance of a prediction rule customized for the population which should be reviewed appropriately.

\section{Limitations}

The required sample size for the infected patients was not achieved during the study period even after extending the enrolment of subjects beyond the required 61 noninfected patients which can affect the results of the study and the generalizability of the results. Disease severity measure and other methods of diagnosis of invasive candida infection such as eye assessment, ultra-sound scanning were not utilized due to feasibility issues. Use of a wide range of adult, paediatric and oncological ICUs setting may have affected the study of risk factors.

\section{Conclusions}

Combination of CS which had excellent sensitivity with corrected CI which had good specificity could reliably predict candidaemia in our study sample.

\section{Acknowledgements}

We would like to thank UGC and Faculty Research Committee for providing funds for the study, Dr P Kalukottege, Consultant Microbiologist for providing CLSI documents, Dr Gayani Thilakaratne, of Duke University, USA and Dr M Weerasekara, Senior Lecturer of University of Sri Jayawardenapura for generously providing the control strains for Candida quality control, Prof B Perera and Dr Y. Waniganayake for assistance with statistical methods, the staff of Department of Microbiology of Faculty of Medicine, University of Ruhuna for the technical assistance.

\section{Conflicts of Interest}

Authors declare no conflicts of interest.

\section{Abbreviations}

CI - Candida colonization Index

CLSI - Clinical and Laboratory Standards Institute

CCI - Corrected Candida colonization Index

CS - Candida Score

ICU - Intensive Care Unit

\section{References}

1. Arendrup MC. Epidemiology of invasive Candidiasis. Curr Opin Crit Care 2010; 16: 445-52.

2. Kullberg BJ, Arendrup MC. Invasive Candidiasis. $N$ Engl J Med 2015; 373: 1445-56.

3. Pfaller MA, Diekema DJ. Epidemiology of invasive Candidiasis: a persistent health problem. Clin Microbiol Rev 2007; 20: 133-63.

4. Lortholary O, Renaudat $\mathrm{C}$, Sitbon $\mathrm{K}$, et al. Worrisome trends in incidence and mortality of candidemia in intensive care units (Paris area, 2002-2010). Intensive Care Med 2014; 40: 1303-12.

5. Pittet D, Eggimann P. Candida colonization and subsequent infections in critically ill surgical patients: 20 years later. Intensive Care Med 2014; 40: 1429-48.

6. Leon C, Ruiz-Santana S, Saavedra P, et al. Abedside scoring system ("Candida Score") for early antifungal treatment in non neutropaenic critically ill patients with Candida colonization. Crit Care Med 2006; 34: 730-7.

7. Eggimann $\mathrm{P}$, Que Y, Revelly, J, et al. Preventing invasive Candida infections. Where could we do better? J Hosp Infect 2014; 89: 302-8.

8. Pittet D, Monod M, Suter PM, et al. Candida colonization and subsequent infections in critically ill surgical patients. Ann Surg 1994; 220: 751-8.

9. Antonysamy B, Samuelson P, Christopher S. Biostatistics Principals and Practice. 1st ed. New Delhi, McCrow Hill. 2010. 
10. Lau AF, Kabir M, Sharon C, et al. Candida colonization as a risk marker for invasive Candidiasis in mixed medical and surgical ICUs: development and evaluation of a simple standard protocol. J Clin Microbiol 2015; 53: 1324 30 .

11. Caggiano G, Puntillo F, Coretti C, et al. Candida Colonization index in patients admitted to an ICU. Int $J$ Mol Sci 2011; 12: 7038-47.

12. Blumberg HM, Jarvis WR, Scoucie M, et al. Risk factors for Candidal blood stream infections in SICU patients: the NEMIS prospective multicentre study. Clin Infect Dis 2001; 33: $177-86$
13. Cuenca-Estrella M, Verweij PE, Arendrup MC, et al. ESCMID guideline for the diagnosis and management of Candida diseases: diagnostic procedures. Clin Microbiol Infect 2012; 18: 9-18.

14. Leon C, Ruiz-Santana S, Saavendra P, et al. Usefulness of Candida score for discriminating between Candida colonization and invasive Candidiasis in non-neutropaenic critically ill patients: a prospective multicentre study. Crit Care Med 2009; 37: 1624-33.

15. Ahmed A, Azim A, Baronia AK, et al. Risk prediction for invasive Candidiasis. Indian J Crit Care Med 2014; 18: 682-8. 